\title{
Successful tongue cancer surgery under general anesthesia in a 99-year-old patient in Okinawa, Japan: A case report and review of the literature
}

\author{
TESSHO MARUYAMA ${ }^{1,2}$, TOSHIYUKI NAKASONE ${ }^{2}$, AKIRA MATAYOSHI ${ }^{2}$ and AKIRA ARASAKI ${ }^{1,2}$ \\ ${ }^{1}$ Department of Oral and Maxillofacial Functional Rehabilitation, Graduate School of Medicine, University of the Ryukyus; \\ ${ }^{2}$ Department of Clinical Neuroscience, Oral and Maxillofacial Functional Rehabilitation, \\ University of the Ryukyus Hospital, Nishihara, Okinawa 903-0215, Japan
}

Received February 13, 2015; Accepted April 15, 2016

DOI: $10.3892 / \mathrm{ol} .2016 .4864$

\begin{abstract}
As advances in the medical field have resulted in increased life expectancy, performing surgery under general anesthesia in elderly patients has become an important issue. A 99-year-old Okinawan female was admitted to the hospital presenting with pain in the tongue. Following physical examination, a clinical diagnosis of early stage tongue cancer (T2NOMx) was confirmed. Early stage tongue cancer is particularly easy to access for surgical resection. By contrast, later stages of tongue cancer are associated with pain, dysphagia and throat obstruction. The patient and their family agreed to surgery due to the worsening pain associated with the tumor and gave informed consent for surgery. Following consultation with a cardiologist and an anesthesiologist, the tongue tumor was surgically resected under general anesthesia. Subsequent to surgery, the patient experienced pain relief and was discharged from the hospital on day 14 post-surgery. The patient was able to maintain the same quality of life, and lived for 5 years and 2 months longer without evidence of disease, surviving to the age of 104 years old. The present case demonstrates that surgery under general anesthesia may be appropriate in patients of an advanced age, with a treatment plan that should ideally be based on careful assessment of the wishes of the patient and their family, medical risks, and benefits and economic costs of alternative treatments, in addition to consideration of the patient's culture.
\end{abstract}

\section{Introduction}

As life expectancy continues to increase, the number of elderly patients requiring surgery for the treatment of cancer

Correspondence to: Professor Akira Arasaki, Department of Oral and Maxillofacial Functional Rehabilitation, Graduate School of Medicine, University of the Ryukyus, 207 Uehara, Nishihara, Okinawa 903-0215, Japan

E-mail: arasaki@med.u-ryukyu.ac.jp

Key words: tongue cancer, general anesthesia, elderly also increases $(1,2)$. Cancer management of nonagenarian or centenarian patients is challenging. Although maintenance of good health is necessary to achieve longevity, organ function will deteriorate over time, making the elderly susceptible to stress, including trauma or surgery, which compromises homeostasis and results in organ dysfunction or aggravates existing dysfunction $(1,2)$. Elderly patients have increased morbidity and mortality risks; thus, balancing higher toxicity risks with potential improvement of quality of life due to anticancer therapies presents a significant challenge when caring for particularly elderly patient (3).

In 2014, the population of Japan included $>60,000$ citizens aged $\geq 100$ years (4). Japan has an increasing elderly population, with Okinawa in particular having one of the highest proportion of elderly individuals. In the Okinawan culture, a preference for noninvasive care for elderly patients with cancer may present challenging circumstances for clinicians when the cancer may be curable (3). Palliative care may not always be a sufficient treatment choice, particularly if patients suffer from impaired quality of life and pain from symptoms of untreated cancer progression (3). Early stage tongue cancer is particularly easy to access for surgical resection. By contrast, if left untreated, later stages of this disease is associated with pain, dysphagia and throat obstruction. Therefore, the optimal treatment for early stage oral cavity squamous cell carcinoma (SCC) is surgery (5).

The current case describes a 99-year-old patient with early stage tongue cancer, which was successfully resected under general anesthesia. The patient lived disease-free for 5 years, surviving to 104 years old. The study was conducted in compliance with the Declaration of Helsinki and was approved by the Ethics Committee of the University of The Ryukyus (Okinawa, Japan). Written informed consent was obtained from the next of kin of the patient for publication of the present case report and any accompanying images.

\section{Case report}

A 99-year-old female, who was independent in her daily activities, presented to our hospital with a 2-month history of pain at the right edge of the tongue. The patient had no 
history of trauma to the tongue. Oral examination identified an indurated, ulcerated mass, measuring $3 \times 1.5 \mathrm{~cm}$ in diameter and was located in the right tongue margin (Fig. 1). There was no restriction of tongue movement and no palpable cervical lymph nodes. A clinical diagnosis of early stage tongue cancer (T2N0Mx) was made according to the Union for International Cancer Control TNM classification system (6). A computed tomography (CT) scan was performed following administration of intravenous contrast material and identified an enhancing tumor measuring $1.3 \times 0.7 \mathrm{~cm}$ in the right margin of the tongue.

On examination, the patient was $1.39 \mathrm{~m}$ tall and weighed $37 \mathrm{~kg}$ with a body mass index of $19.15 \mathrm{~kg} / \mathrm{m}^{2}$. By surgical considerations, the patient was New York Heart Association class I and American Society of Anesthesiologist Physical Status 2. Following a number of tests, it was noted that the patient's blood pressure, pulse rate, respiratory rate and $\mathrm{SaO}_{2}$ were within the normal ranges $(124 / 70 \mathrm{mmHg}, 74$ beats/min, 18 breaths/min and $98 \%$ in room air, respectively). The patient had a history of cerebral infarction 10 years prior to presentation, in addition to hypertension, sinus bradycardia and kyphosis with a thoracic compression fracture, but demonstrated no history of hyperlipidemia, diabetes, cardiac arrhythmia or other evidence of heart disease. There were no other significant findings from physical examination. A surgical treatment strategy was discussed with the patient and the family, and informed consent was obtained from the next of kin.

Preoperative blood and urine tests reported normal blood counts, electrolytes and blood coagulation results. Bilirubin, urea and creatinine levels were unchanged following diagnostic exams and surgery. The total protein of the patient was $6.9 \mathrm{~g} / \mathrm{dl}$. An X-ray of the chest was normal, and an electrocardiogram reported the presence of sinus bradycardia. The patient was evaluated by a cardiologist and an anesthesiologist prior to selecting a treatment strategy. An echocardiogram revealed moderate pulmonic and tricuspid regurgitation, which the cardiologist assessed as age-appropriate, and a normal ejection fraction of $78 \%$. A CT scan of the brain identified no new cerebral infarctions. The anesthesiologist determined that the patient was a good candidate for general anesthesia, and suggested that kyphotic surgical task curvature should be utilized to modify the supine surgical position due to kyphosis (Fig. 2).

Surgical resection of the tongue tumor was performed, and $10 \mathrm{~mm}$ tumor-free margins were obtained (Fig. 3). Following resection, TERUDERMIS ${ }^{\circledR}$ (Terumo Corporation, Tokyo, Japan), a collagen-based artificial dermis, was grafted onto the cleansed wound surface. The surgical procedure lasted $60 \mathrm{~min}$ in the absence of hypotension, bradycardia or decreased oxygen saturation (2). Histopathology of the tongue mass specimen revealed moderate proliferation of SCC cells. Inflammation was noted beneath the epithelium (Fig. 4), but peripheral infiltration of the tumor cells was not observed. Thus, the lesion was diagnosed as SCC.

The postoperative course was complicated by slight pain, but the patient did not experience any episodes of delirium. Nonsteroidal anti-inflammatory drugs were administered for pain relief, either as a suppository or internally. Intravenous hydration was discontinued on the first postoperative day and oral feeding was resumed $6 \mathrm{~h}$ post-surgery. The patient was hospitalized for 14 days for routine monitoring due to old age. Subsequent to being discharged, the patient lived for 5 years and 2 months more without any evidence of disease recurrence (Fig. 5).

\section{Discussion}

Living to 100 years of age is no longer a rarity (2), with $>60,000$ centenarians living in Japan. Okinawa is a prefecture located in the southernmost part of Japan and is well-known around the world for the longevity of its populations. According to statistical reports from the World Health Organization and the Japanese Ministry of Health, Labour and Welfare, Okinawan individuals experience extremely low mortality from stroke, coronary artery disease, and hormone-dependent cancer of the ovary, prostate and breast (7). It has also been reported that Okinawan residents who follow a traditional lifestyle of physical exercise, diet, cooking, stress coping, guidance of the 'ikigai' (meaning living satisfaction) and food culture, enjoy extended longevity $(7,8)$. Despite rapid changes in regional politics and lifestyle, the people of Okinawa maintain cultural respect of their ancestry and the elderly with close family ties (9).

In general, advances in the medical field have resulted in increases in life expectancy. Therefore, surgery with general anesthesia has become an important issue for elderly patients (2). Aging is associated with various physiological changes that affect functional reserve and result in a diminished ability to respond appropriately to stresses such as surgery (2). Perioperative mortality rates have decreased in elderly patients, however, the long-term benefit to these individuals is unknown (10). The National Cancer Center Network (NCCN) has published a series of guidelines indicating that, in general, age is not the primary consideration for surgical risk (11). Although surgery under general anesthesia in nonagenarians and centenarians is not without risk, available studies regarding surgery in centenarians suggest that there is no significantly increased risk of mortality (12-15). Results of a previous study of nonagenarians and centenarians support the hypothesis that cardiac surgical procedures may appropriately be performed on a higher-risk patient group independent of age (16). The same study also demonstrated that the majority of nonagenarians and centenarians could be preoperatively identified as having a relatively low mortality risk $(2,16)$. A recent study of 1,201 patients undergoing major surgery for head and neck cancer identified that surgical complication rates did not increase with age (17). By contrast, advanced tumor stage and prolonged surgery time were associated with surgical complications (17). Medical evaluation should focus on risk factor identification, optimization of health status, prediction of complications, and providing accurate information to the surgeon, physician and anesthesiologist (2). Although the 99-year-old patient described in the current case presented with evidence of mild clinical diseases, the patient was able to perform normal activities and the laboratory tests were within normal limits.

In 1990, Barzan et al (18) published a retrospective study of 438 elderly patients with head and neck cancer. The study stated that although age did affect several features of patients with head and neck cancer, it did not appear to be 


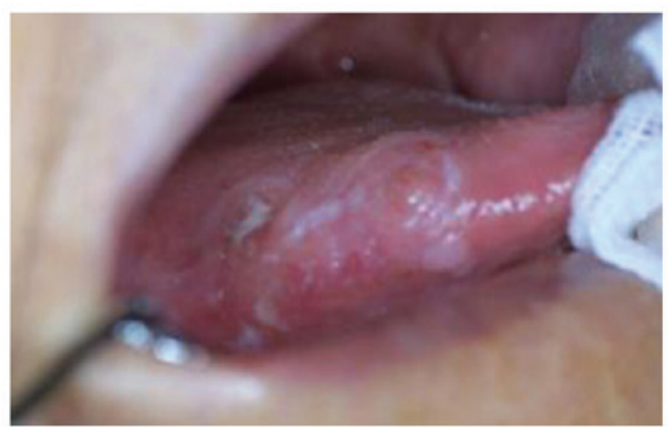

Figure 1. Clinical photograph of right tongue tumor lesion during initial oral examination.

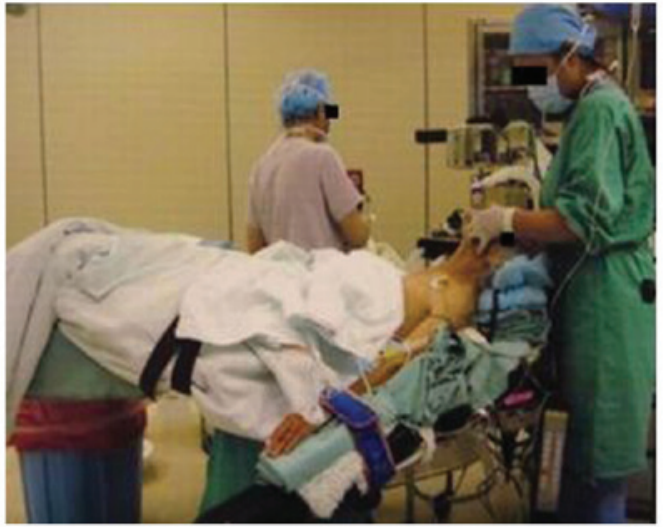

Figure 2. Kyphotic curvature was used intraoperatively to decrease the supine position of the patient due to kyphosis.

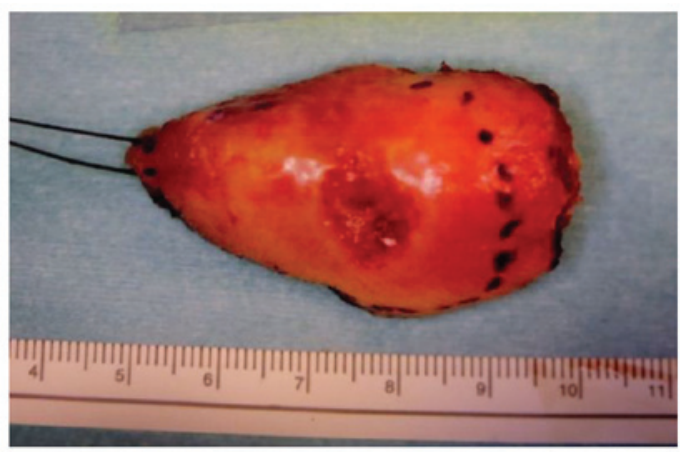

Figure 3. Excised tongue specimen.

an independent prognostic factor for survival and local control (18). Furthermore, the study outlined the treatment policy for head and neck cancer during those years as follows: All resectable lesions underwent radical surgery, followed by radiotherapy if the resected tumor demonstrated unfavorable histological prognostic features, including extra-nodal spread or positive surgical resection margins (18). Radiotherapy alone, or combined with chemotherapy, was administered to patients with loco-regional tumor extension or poor status that precluded radical surgery (18).

Older patients with head and neck cancer require more supportive care (19). The NCCN guidelines also emphasize the application of supportive care to minimize toxicity and promote

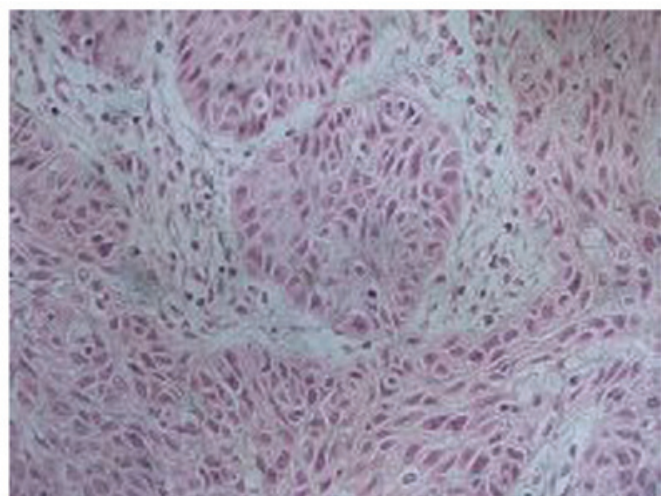

Figure 4. Tumor specimen showing moderate proliferation of squamous cell carcinoma cells. Staining, hematoxylin and eosin; magnification, x400.

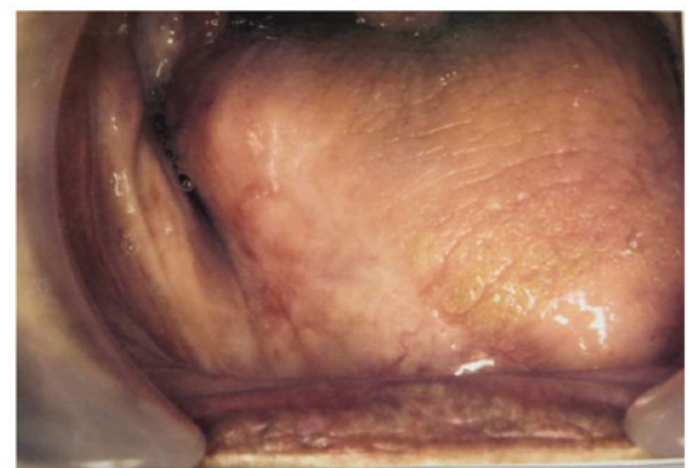

Figure 5. Clinical photograph 2 years post-surgery.

cancer treatment effectiveness among older patients (11). The preanesthetic evaluation of the entire procedure and projected rate of postoperative recovery allows for successful surgical treatment (2). There were no episodes of postoperative delirium. Prehospital and postoperative nutritional status may also serve an important role in the surgical outcomes for centenarians $(2,20)$, and thus, in the present case, oral feeding was resumed $6 \mathrm{~h}$ post-surgery. Furthermore, a coordinated care approach between surgical and geriatric medical teams is highly desirable to achieve positive outcomes $(15,21)$.

In the current case, the patient and their family agreed to the performance of surgical resection under general anesthesia, which provided pain relief and allowed the patient to maintain a good quality of life. The most notable fact of the present case is perhaps that the patient was discharged without complications and lived without recurrence for 5 more years. To the best of our knowledge, this patient is one of the oldest individuals to undergo a partial tongue cancer resection under general anesthesia. Despite previous studies describing the treatment of head and neck cancer in elderly patients (22-24), a previous study describing the treatment of tongue cancer by surgery under general anesthesia in a patient $\geq 99$ years old could not identified.

In conclusion, a treatment plan should ideally be based on careful assessment of the wishes of the patient and their family, medical risks, and benefits and economic costs of alternative treatments (2), in addition to consideration of the patient's culture. The present case demonstrates that an elderly patient 
is able to undergo successful surgical resection under general anesthesia. The relative safety of surgical interventions and perioperative care of elderly patients should encourage physicians to keep surgical treatment options in mind despite their advanced age.

\section{Acknowledgements}

The authors would like to thank Enago (www.enago.jp) for an English language review of the original manuscript.

\section{References}

1. Rosenthal RA and Kavic SM: Assessment and management of the geriatric patient. Crit Care Med 32 (Suppl 4): S92-S105, 2004.

2. Imbelloni LE, Lima U and Pedrosa FK: Successful anesthesia and hip surgery in a 107-year-old patient. Am J Case Rep 15: 308-311, 2014

3. Chien CR and Shih YC: Use of personalized decision analysis in decision making for palliative vs. surgical management of the oldest-old patients with localized skin cancer in a culturally sensitive environment: A case study of a 96-year-old male Taiwanese patient. J Pain Symptom Manage 45: 792-797, 2013.

4. Japan Statistics Bureau: Japan Population Statistics. http://www. stat.go.jp/data/jinsui/pdf/201407.pdf AccessedJuly 22, 2014 (In Japanese).

5. Shah JP: Surgical approaches to the oral cavity primary and neck. Int J Radiat Oncol Biol Phys 69 (Suppl 2): S15-S18, 2007.

6. Sobin LH, Gospodarowicz MK and Wittekind C: Head and Neck tumours. In: TNM Classification of Malignant Tumours. O'Sullivan B (ed.). 7th edition. Wiley-Blackwell, Oxford, pp22-62, 2009.

7. Suzuki M, Willcox B and Willcox C: Successful aging: Secrets of okinawan longevity. Geriatr Gerontol Int 4 (s1): S180-S181, 2004

8. Sho H: History and characteristics of Okinawan longevity food. Asia Pac J Clin Nutr 10: 159-164, 2001.

9. Iseki K: Screening and prevention of renal disease: Large population study in Okinawa, Japan. Nephrology 4: S86-S89, 1998.

10. Clayman GL, Eicher SA, Sicard MW, Razmpa E and Goepfert H: Surgical outcomes in head and neck cancer patients 80 years of age and older. Head Neck 20: 216-223, 1998.
11. National Comprehensive Cancer Network: NCCN Clinical Practice Guidelines in Oncology (NCCN Guidelines): Older Adult Oncology, 2015. Available from: http://www.nccn.org/ professionals/physician_gls/pdf/senior.pdf. Accessed January 15, 2015.

12. Forster MC and Calthorpe D: Mortality following surgery for proximal femoral fractures in centenarians. Injury 31: 537-539, 2000.

13. Warner MA, Saletel RA, Schroeder DR, Warner DO, Offord KP and Gray DT: Outcomes of anesthesia and surgery in people 100 years of age and older. J Am Geriatr Soc 46: 988-993, 1998.

14. Katlic MR: Surgery in centenarians. JAMA 253: 3139-3141, 1985.

15. Ozorio G and Chan DK: Surgery in the very elderly. Intern Med J 37: 837-838, 2007.

16. Bridges CR, Edwards FH, Peterson ED, Coombs LP and Ferguson TB: Cardiac surgery in nonagenarians and centenarians. J Am Coll Surg 197: 347-357, 2003.

17. Peters TT, van Dijk BA, Roodenburg JL, van der Laan BF and Halmos GB: Relation between age, comorbidity and complications in patients undergoing major surgery for head and neck cancer. Ann Surg Oncol 21: 963-970, 2014.

18. Barzan L, Veronesi A, Caruso G, Serraino D, Magri D, Zagonel V, Tirelli U, Comoretto R and Monfardini S: Head and neck cancer and ageing: A retrospective study in 438 patients. J Laryngol Otol 104: 634-640, 1990.

19. VanderWalde NA, Fleming M, Weiss J and Chera BS: Treatment of older patients with head and neck cancer: A review. Oncologist 18: 568-578, 2013.

20. Takeda S, Noji H, Hirose N, Arai Y, Yamamura K, Shimizu K, Homma S, Ebihara Y and Takayama M: Nutritional intake by the oldest elderly Japanese. Tokyo Centenarian Study 6. Nippon Ronen Igakkai Zasshi 35: 548-558, 1998 (In Japanese).

21. Solomon DH: A statement of principles: Toward improved care of older patients in surgical and medical specialities. J Am Geriatr Soc 48: 699-701, 2000.

22. Siddiqui $\mathrm{F}$ and Gwede CK: Head and neck cancer in the elderly population. Semin Radiat Oncol 22: 321-333, 2012.

23. Syrigos KN, Karachalios D, Karapanagiotou EM, Nutting CM, Manolopoulos L and Harrington KJ: Head and neck cancer in the elderly: An overview on the treatment modalities. Cancer Treat Rev 35: 237-245, 2009.

24. Sarris EG, Harrington KJ, Saif MW and Syrigos KN: Multimodal treatment strategies for elderly patients with head and neck cancer. Cancer Treat Rev 40: 465-475, 2014. 